
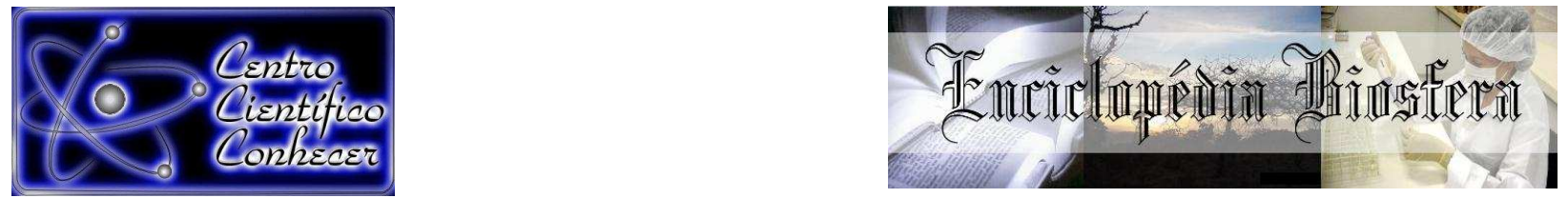

\title{
CONCENTRAÇÃO DAS ATIVIDADES ANTIOXIDANTES EM PLANTAS DE JAMBU, CULTIVADAS SOB ADUBAÇÃO ORGÂNICA E MINERAL
}

\footnotetext{
Luciana da Silva Borges ${ }^{1}$, Rumy Goto $^{2}$, Kelly de Nazaré Maia Nunes ${ }^{3}$, Fabio Vianello ${ }^{4}$, Giuseppina Pace Pereira Lima ${ }^{5}$

${ }^{1}$ Professora Doutora, UFRA-Campus Paragominas, endereço: rodovia PA 256, KM 06, S/N, Nova Conquista, Paragominas, CEP: 68627451. Email:

luciana.borges@ufra.edu.br*Autor para correspondência

${ }^{2}$ Professora, Doutora, Departamento de Produção Vegetal, Setor Horticultura,

Faculdade de Ciências Agronômicas, Universidade Estadual Paulista "Júlio de Mesquita Filho" (UNESP), Botucatu, São Paulo, Brasil.

${ }^{3}$ Doutoranda em Agronomia/Horticultura da Universidade Estadual Paulista "Júlio de

Mesquita F ilho" (UNESP), Botucatu, São Paulo, Brasil.

${ }^{4}$ Professor do Doutor do Departamento de Química, Universidade de Pádua, Itália.

${ }^{5}$ Professora, Doutora, Departamento de Química e Bioquímica, Instituto de

Biociências, Universidade Estadual Paulista "Júlio de Mesquita Filho" (UNESP).

Distrito de Rubião Junior S/N, Botucatu, São Paulo, Brasil

\section{Recebido em: 08/09/2015 - Aprovado em: 14/11/2015 - Publicado em: 01/12/2015} DOI: http://dx.doi.org/10.18677/Enciclopedia_Biosfera_2015_118
}

\begin{abstract}
RESUMO
O jambu (Spilanthes oleracea L.) é uma planta rica em metabolitos secundários tais como o espilantol, trans-cariofileno, germacreno D, L-dodeceno e espatulenol, que são responsáveis pelas bioatividades anti-inflamatória, anti-sépticas e anestésica da espécie. Essa qualidade farmacológica torna essa hortaliça promissora para obtenção de substâncias químicas para as indústrias de cosméticos e farmacológica. Realizou-se essa pesquisa com o objetivo de verificar se o modo de cultivo orgânico influencia o nível de antioxidantes em folha e inflorescência de jambu. O delineamento estatístico foi em blocos casualizados, fatorial $(2 \times 2)$, sendo duas adubações (orgânica e mineral) e duas colheitas (aos 90 dias após a semeadura e 40 dias após a rebrota), com 4 repetições. As plantas foram separadas em folhas e inflorescências, congeladas em nitrogênio líquido e armazenadas em freezer a $-80^{\circ} \mathrm{C}$. Analisou-se o teor de fenois totais, flavonoides totais, vitamina $\mathrm{C}$ e o potencial antioxidante através de três diferentes métodos. Os dados obtidos foram analisados estatisticamente através da análise de variância, com teste $F$. Estabeleceu-se o coeficiente de correlação de Pearson, entre todas as características analisadas. A adubação mineral foi eficaz, quanto aos teores de flavonoides em jambu, sendo as folhas o principal local de produção e foi eficaz também na produção de fenóis totais em inflorescências. A vitamina $C$ ocorreu em maior teor somente na primeira colheita de plantas de origem orgânica. Para determinação da concentração de polifenois para planta de jambu o método enzimático foi o que apresentou maior atividade.
\end{abstract}

PALAVRAS-CHAVE: Fenois, Flavonoides, método enzimático, Spilanthes oleracea, Vitamina C 


\title{
CONCENTRATION OF ANTIOXIDANT ACTIVITY IN PLANTS OF JAMBU, CULTIVATING UNDER ORGANIC AND MINERAL FERTILIZATION
}

\begin{abstract}
The Jambu (Spilanthes oleracea L.) is a plant rich in secondary metabolites such as espilantol, trans-caryophyllene, germacrene D, L-dodecene and spathulenol, which are responsible for the anti-inflammatory bioactivities, antiseptic and the anesthetic species. This pharmacological quality makes this promising vegetable to obtain chemicals for the cosmetics and pharmaceutical industries. This research in order to verify whether organic cultivation methods influences the level of antioxidants in leaf and inflorescence of Jambu took place. The experimental design was a randomized block factorial $(2 \times 2)$, two fertilizer (organic and mineral) and two samples (at 90 days after sowing and 40 days of regrowth), with four repetitions. The plants were separated into leaves and flowers, frozen in liquid nitrogen and stored in a freezer at $-80^{\circ} \mathrm{C}$. It analyzed the content of total phenols, total flavonoids, vitamin $\mathrm{C}$ and the antioxidant potential by three different methods. Data were statistically analyzed by ANOVA with $F$ test was established the Pearson correlation coefficient between all characteristics analyzed. The mineral fertilizer was effective, as the flavonoid content in Jambu, the leaves being the main production site and was also effective in the production of phenolic compounds in inflorescences. Vitamin C content was at most only the first harvest of organic origin plants. For determining the concentration of polyphenols to jambu plant enzymatic method it showed the highest activity.
\end{abstract}

KEYWORDS: phenols, flavonoids, enzymatic method, Spilanthes oleracea, vitamin C

\section{INTRODUÇÃO}

O jambu (Spilanthes oleracea L.) é uma planta nativa da Amazônia, de clima tropical e segundo HOMMA et al. (2011), é uma espécie da família Asteraceae. Essa olerácea é produzida e consumida na região Norte do Brasil, principalmente no Pará, sendo sua maior demanda nos períodos festivos, tais como o Círio de Nazaré e as festas de fim de ano, para ser ultilizado como condimento na culinária regional. Popularmente essa planta também é utilizada como erva medicinal, pois segundo os dizeres populares suas folhas e flores podem ser recomendadas para elaboração de infusões no tratamento de anemia, dor de dente e garganta, sendo sugerido como antibiótico e anestésico (BORGES et al., 2013a). Outra forma de consumo do jambu, principalmente em São Paulo é em restaurantes de comidas exóticas, onde as inflorências são utlizadas para decorar os pratos diferenciados dos restaurantes (BORGES et al., 2014a).

Relatos de LORENZI \& MATOS (2002), indicam que a planta de jambu possui em torno de $0.7 \%$ de óleo essencial, na sua composição química. E apresenta na cv. Jambuarana, um total de 66 substâncias (BORGES et al., 2014b). Segundo BORGES et al. (2013a) e BORGES et al. (2012) dentre essas substâncias, tem-se o espilantol, trans-cariofileno, germacreno D, L-dodeceno e espatulenol, características bioquímicas essas que vem atraindo a atenção de empresas farmacêuticas e de cosméticos para as plantas de jambu. LI-CHEN et al. (2008) realizando a análise da substância espilantol concluiram que esse composto possui efeito anti-inflamatório. Sendo assim esses autores recomendaram manipular essa substância para desenvolvimento de drogas antiinflamatórias.

Além das propriedades farmacológicas, estão presentes na composição 
substâncias antioxidantes benéficas a saúde humana, entre estas os compostos fenólicos, vitamina $\mathrm{C}$ e carotenoides, que podem ajudar a proteger as células contra os danos da oxidação causados pelos radicais livres no organismo.

$\mathrm{Na}$ planta, a qualidade e alterações dos antioxidantes podem ser induzidos por fatores de estresse biótico ou abióticos, como por exemplo, ataque de patogénos, tipos de adubação, deficiência nutricional, baixa temperatura e danos mecânicos. Em função disso as plantas podem modificar o metabolismo de compostos fenólicos e da capacidade antioxidante como um mecanismo para proteger contra a mencionada tensões (DIXON \& PAIVA, 1995).

Os principais antioxidantes dietéticos são algumas vitaminas, compostos fenólicos e carotenoides. Os antioxidantes agem interagindo com os radicais livres antes que estes possam reagir com as moléculas biológicas, evitando que ocorram as reações em cadeia ou prevenindo a ativação do oxigênio a produtos altamente reativos (BERNARDES et al., 2010).

A preferência no mercado por produtos que contenham alto teor de antioxidantes está muitas vezes aliada à ideia de que estão livres de muitos agrotóxicos capazes de induzir uma série de doenças na população, que podem ser desencadeadas pelos organoclorados, fosforados, carbamatos, etc., ou seja, um coquetel de pesticidas que são utilizados de maneira incorreta e abusiva na agricultura convencional (JANSSEN \& HAMM, 2012). Geralmente, parte dessa população devido a esse conceito formado, prefere os produtos de origem orgânica.

No Brasil, o sistema orgânico de produção está regulamentado pela Lei Federal no 10.831, de 23 de dezembro de 2003, que contém normas disciplinares para a produção, tipificação, processamento, envase, distribuição, identificação e certificação da qualidade dos produtos orgânicos. O Brasil é forte na produção orgânica de açúcar, soja, café, amêndoas, mel, frutas e olerícolas para o mercado interno (MAPA, 2012). O mercado mundial de orgânicos já movimenta, anualmente, mais de US $\$ 40$ bilhões, com crescimento médio de $25 \%$ ao ano (SGANZERLA; MARTINS \& SINGH, 2013), entretanto, poucos estudos têm sido conduzidos em relação à plantas que tem potencial antioxidante, ou seja, até mesmo farmacológico.

$\mathrm{Na}$ agricultura orgânica, nutrientes são fornecidos através de rotação de culturas, plantas de cobertura, e esterco animal. O impacto de agricultura biológica sobre a qualidade dos produtos agrícolas ainda permanece em discussão, devido a uma falta de dados técnicos (PICCHI et al., 2012). Segundo BORGES et al. (2013b) cada vez mais a população esta preferindo hortaliças de cultivo orgânico, pois estas estão livres de agroquímicos, e para esses mesmo autores aliado a isso está fato dos pequenos produtores trabalharem basicamente com adubos orgânicos em detrimento aos adubos sintéticos por dificuldade de aquisição,. Sendo isso uma realidade da região norte, onde tem maior área de cultivo da planta de jambu. Dessa forma, essa pesquisa teve o objetivo de analisar o teor de polifenois e atividade antioxidante em folha e inflorescências de jambu, cultivado na adubação orgânica e mineral.

\section{MATERIAL E MÉTODOS}

\section{Obtenção da planta}

O experimento foi conduzido na Fazenda Experimental São Manuel (São Manuel-SP), pertencente a Faculdade Ciências Agronômicas, Universidade Estadual Paulista "Júlio de Mesquita Filho", Campus de Botucatu, localizada no município de São Manuel, sob as coordenadas geográficas de latitude $22^{\circ} 44^{\prime} 50^{\prime \prime}$ Sul e longitude 
48 $34^{`} 00^{\prime \prime}$ Oeste de Greenwich, com altitude em tor no de $765 \mathrm{~m}$. O clima da região, segundo ESPINDOLA et al. (1974), é do tipo mesotérmico, Cwa (subtropical úmido com estiagem no período de inverno).

\section{Cultivo do jambu}

As plantas de jambu foram cultivadas em campo aberto, no qual a semeadura foi realizada em bandejas de poliestireno expandido de 128 células, contendo o substrato comercial Plantmax®. Utilizando-se cinco sementes de Jambu cv. Jambuarana, em cada célula. A emergência ocorreu aos sete dias, sendo realizado o desbaste deixando uma plântula por célula. $O$ transplante foi realizado aos 40 dias após a semeadura, manualmente, quando as mudas apresentavam-se com seis folhas definitivas, em quatro canteiros de $6 \mathrm{~m}^{2}$, colocando-se 18 plantas por linha e cada canteiro constou de cinco linhas. O espaçamento utilizado foi de $20 \times 25 \mathrm{~cm}$. As capinas foram realizadas a cada dez dias, desde o início da instalação da cultura.

O delineamento experimental utilizado foi em blocos casualizados em esquema fatorial ( $2 \times 2$ ), sendo duas adubações (orgânica e mineral) e duas colheitas (aos 90 dias após a semeadura e 40 dias após a rebrota), com 4 repetições.

Para adubação orgânica aplicou-se $8 \mathrm{~kg} / \mathrm{m}^{-2}$ de esterco de curral no plantio e para adubação em cobertura foram realizadas aplicações parceladas de $1 \mathrm{~kg} / \mathrm{m}^{2}$ de torta de mamona aos 55, 70 e 80 dias após o transplante de plantas de jambu. Para a adubação mineral utilizou-se $120 \mathrm{~g} / \mathrm{m}^{-2}$ de nitrato de amônia, $200 \mathrm{~g} / \mathrm{m}^{-2}$ de superfosfato simples e $50 \mathrm{~g} / \mathrm{m}^{-2}$ de cloreto de potássio no plantio e para a adubação em cobertura aplicou-se $50 \mathrm{~g} / \mathrm{m}^{-2}$ de NPK na formulação de $(15,15,20)$, sendo 15 partes de nitrogênio $(N), 15$ partes de fósforo $(P)$ e 20 partes de potássio $(K)$, aos 55 , 70 e 80 dias após o transplante.

Ao final ciclo foi realizada a colheita, nas primeiras horas do dia, aos 90 dias após a semeadura,sendo considerado o ponto de colheita a abertura do botão floral. Os ramos foram cortados a sete $\mathrm{cm}$ do solo. Com relação a segunda colheita, essa foi realizada aos 40 dias, após a primeira colheita.

As plantas foram lavadas em água para retirada das impurezas e mantidas por 5 min em solução de $150 \mathrm{mg}^{-1}{ }^{-1}$ de cloro ativo, visando reduzir a contaminação microbiana do vegetal. Para a retirada de possível contaminação por cloro, foram realizadas diversas lavagens em água destilada. Parte do material vegetal foi seco em estufa para análise do teor de fenóis totais. Após a retirada do excesso de água, folha e inflorescência foram congeladas em nitrogênio líquido e armazenadas em freezer a $-80^{\circ} \mathrm{C}$. As amostras foram pulverizadas em nitrogênio líquido e submetidas a analises bioquímicas.

\section{Teor de fenois totais}

A análise de fenóis totais foi realizada de acordo com o método espectrofotométrico com o uso do reativo de Folin-Ciocalteu (SINGLETON \& ROSSI Jr., 1965). Amostras do material seco e moído foram pesadas e colocadas em tubos de centrífuga, contendo acetona $50 \%$. Em seguida foram levados para banho ultrassônico por 20 min e posteriormente centrifugados a $6.000 \mathrm{rpm}$ (HETTICH ZENTRIFUGEN MIKRO 220R) durante $10 \mathrm{~min}$ e o sobrenadante foi recolhido. 0 precipitado foi re-extraído e os sobrenadantes combinados. Alíquotas de $0,1 \mathrm{~mL}$ do sobrenadante foram transferidas para tubos de ensaio, juntamente com $0,5 \mathrm{~mL}$ do reagente Folin-Ciocalteau e 2,5 mL de solução saturada de $\mathrm{Na}_{2} \mathrm{CO}_{3}$. Após 1 hora de reação (completa precipitação do carbonato) a leitura de absorbância foi realizada a 
725 ๆm (PHARMACIA BIOTECH ULTROSPEC 2000) e os resultados expressos em $\mu \mathrm{g}$ fenois $\mathrm{g}^{-1}$ massa seca, em equivalente de ácido gálico.

\section{Flavonoides totais}

A extração para análise dos teores dos flavonoides totais foi feita de acordo com o método de AWAD et al. (2000), segundo as adaptações realizadas por POPOVA et al. (2004). Amostras de material fresco foram pesadas e adicionado metanol acidificado 10\%. Posteriormente, foram levadas para banho ultrassônico durante 30 min e adicionado cloreto de alumínio 5\% e centrifugadas por 20 min a $10000 \mathrm{rpm}$ (JOUAN MR 18 12). Em seguida, as amostras foram filtradas e a leitura de absorbância realizada a $425 \mathrm{\eta m}$. Os resultados foram expressos em $\mu \mathrm{g}$ flavonoides $\mathrm{g}^{-1}$ massa fresca, em equivalente de rutina.

\section{Teor de vitamina $\mathbf{C}$}

A determinação do ácido ascórbico foi feita segundo método de TERADA et al. (1978).

\section{Determinação da atividade antioxidante por três métodos}

Método pelo DPPH• (2,2-difenil-1- picril-hidrazil)

Foi utilizada a metodologia de BRAND-WILLIAMS et al. (1995). A solução de DPPH• foi preparada a 2,10 $\mathrm{g} \mathrm{mL}^{-1}(0,0100 \mathrm{mg}$ de DPPH em $50 \mathrm{~mL}$ de etanol a $99,8 \%$ ). Para a extração foram pesados $0,300 \mathrm{~g}$ da amostra fresca de jambu e diluídas em $10 \mathrm{~mL}$ de etanol a 99,8\% em tubo para centrífuga. As amostras foram centrifugadas a $2.000 \mathrm{rpm}$ (HETTICH ZENTRIFUGEN MIKRO 220R) por $10 \mathrm{~min}$ a $5^{\circ} \mathrm{C}$. Alíquota de $0,500 \mu \mathrm{L}$ do sobrenadante foi combi nado com $3 \mathrm{~mL}$ de etanol P.A. Adicionados $300 \mu \mathrm{L}$ de DPPH $2 \times 10^{-4} \mathrm{~g} \cdot \mathrm{mL}^{-1}$, após a homogeneização, os tubos de ensaios foram armazenados no escuro por $60 \mathrm{~min}$. Um controle negativo foi feito com o DPPH a 0,3 mM em etanol para observar o decaimento do radical contra os antioxidantes doadores. A leitura obtida a $517 \mathrm{~nm}$, foi convertida em porcentagem de atividade antioxidante pela Equação :

$$
\% \text { DPPH reduzido }=\left(\frac{\text { Abs Branco }- \text { Abs Amostra }}{\text { Abs Branco }}\right) \cdot 100
$$

Em que:

Abs Branco: absorvância da solução de DPPH sem a amostra Abs Amostra: absorvância da amostra com o DPPH

Uma curva de calibração foi preparada com 20, 40, 80, 120 e $160 \mu \mathrm{M}$ de BHT e os resultados foram expressos em $\mu \mathrm{M}$ equivalentes de BHT/g amostra (BRANDWILLIAMS et al., 1995).

Método enzimático (ácido linoleico)

A atividade antioxidante via radical peroxil foi realizada a partir de vários extratos etanólicos, por medida da sua capacidade em inibir a peroxidação do ácido linoléico em um sistema micelar (ZENNARO et al., 2007). O método oxigrafico utilizado foi baseado em uma medida cinética que permite obter a capacidade de 
capturar um radical peróxido (PRTC) e a eficiência de captura equivalente radical peróxido (ePRTE) dos extratos. PRTC foi expresso em milimoles de LOO ligado por $1 \mathrm{~g}$ de material fresco e ePRTE foi expresso em milimoles de Trolox em $1 \mathrm{~g}$ de material fresco. A taxa de peroxidação de ácido linoleico foi medida a partir da velocidade de desaparecimento de $\mathrm{O}_{2}$ em um Metrohm 663 VA stand, equipado com um eletrodo de oxigênio Yellow Spring, inserido numa célula oxigrafica termostatizada. $\mathrm{O}$ estudo foi registrado por um computador equipado com uma placa de aquisição de dados (DAQ PCl-6221, série M, Natl. Instruments, Austin, Texas, EUA). O eletrodo de trabalho foi preparado em $-800 \mathrm{mV}$ contra $\mathrm{Ag} / \mathrm{AgCl}$. Os traços oxigraficos experimentais foram processados automaticamente por meio de cálculo para determinar as taxas de consumo de oxigênio a partir dos quais os valores PRTC PRTE foram calculados. A mistura de reação foi preparada por evaporação do solvente a partir de uma solução de 1-lauroilglicerol em diclorometano e por dissolução da película resultante em tampão de fosfato $20 \mathrm{mM}, \mathrm{pH} 7,4$, contendo 5 $\mathrm{mM}$ de sal de sódio do ácido deoxicólico, para se obter $0,5 \mathrm{mM}$ de concentração final de 1-lauroilglicerol. $O$ ácido linoleico (2 $\mathrm{mM}$ de concentração final) foi em seguida adicionado e a solução vigorosamente agitada em vórtice. A solução contendo micelas foi equilibrada com o oxigênio atmosférico na célula a $37 \pm 0,1 \stackrel{\circ}{\circ}$. Após o equilíbrio térmico, ABIP ( $4 \mathrm{mM})$, foi adicionado como uma fonte constante de $\mathrm{LOO}^{\circ}$, que inicia a peroxidação lipídica e os extratos de jambu foram utilizados (concentração final de 0,1-0,01 $\mathrm{g} / \mathrm{mL}$ ) 1 min após a adição ABIP.

\section{Pelo método de Folin-Ciocalteau}

O conteúdo de fenois totais foi medido de acordo com o método de FolinCiocalteu (LEE et al., 2003). As medidas de absorbância foram efetuadas por um espectrofotómetro Varian Cary 50, a $770 \mathrm{~nm}$, após a incubação da mistura de reação durante 60 min à temperatura ambiente. $O$ teor de fenois dos extratos foi expresso em mg de ácido gálico por grama de material fresco.

\section{Índice de atividade antioxidante}

Foi determinado de acordo com SCHERER \& GODOY (2009), onde:

$I A A<0,5=$ pobre atividade antioxidante; $0,5<\mid A A<1,0=$ moderada; $1,0 \leq \mid A A \leq$ $2,0=$ forte $>2,0=$ muito forte.

\section{Análise estatística}

Os dados obtidos foram analisados estatisticamente através da análise de variância, com teste $F$. Quando houve significância para o fator fonte foi aplicado teste de Tukey (5\%) para a comparação de médias. Estabeleceu-se o coeficiente de correlação de Pearson, entre todas as características analisadas, onde as variáveis com coeficientes de correlação positiva e valores abaixo de $0,050 \mathrm{P}$ tendem a aumentar junto. Para os resultados com coeficientes de correlação negativa e valores abaixo de 0,050 $\mathrm{P}$, uma variável tende a diminuir, enquanto a outra aumenta. Para resultados com valores $P$ superiores a 0,050 , não há nenhuma relação significativa entre as duas variáveis.

\section{RESULTADOS E DISCUSSÃO}

A adubação mineral apresentou efeito significativo em relação a adubação orgânica para o teor de flavonoides de folhas (Tabela 1) na primeira, apresentando o valor de 2,60 mg $100 \mathrm{~g} \mathrm{~g}^{-1}$ e segunda colheita, com valores de 2,29 mg $100 \mathrm{~g}^{-1}$ e nas inflorescências, demostrando $5,00 \mathrm{mg} 100 \mathrm{~g}^{-1}$ (Tabela 2) na primeira colheita das plantas de jambu, enquanto que nenhuma influência da adubação é observada no 
teor de fenois totais em folhas. No entanto, o nível de fenois totais aumentou nas inflorescências, apresentando o valor de 460,00 mg $100 \mathrm{~g}^{-1}$, na segunda colheita das plantas cultivadas sob adubação mineral. SMOLEN \& SADY (2009) estudando diversas fontes e formas de aplicação de nitrogênio em cenoura observaram que este mineral altera o teor de compostos fenólicos, sendo que a aplicação de nitrogênio no solo promoveu incrementos no teor dos fenólicos se comparados com a aplicação foliar. Assim, nota-se que a adubação mineral é eficaz, quanto aos teores de flavonoides em jambu, sendo as folhas o principal local de produção e foi eficaz também na produção de fenois totais em inflorescências. No estudo de FALLER \& FIALHO (2009), não ocorreu diferença entre o teor de compostos fenólicos em vegetais cultivados de modo orgânico e mineral. A agricultura orgânica é caracterizada, geralmente, pela restrição do uso de fertilizantes sintéticos e pesticidas. Esta característica tem induzido os pesquisadores a proporem hipóteses, como os orgânicos apresentarem maior conteúdo de polifenolicos (LIMA et al.,2009; ROSSETO, et al., 2015 e LIMA et al., 2014), geralmente induzido por estresse, que por sua vez, induziria a enzima fenilalanina amônia liase (PAL), aumentando assim, o teor de compostos fenólicos. Resultados descritos por GIORGI et al. (2009), evidenciam que a deficiência de nitrogênio comprometeu o metabolismo primário, diminuindo o teor de proteínas, aminoácidos, carotenóides e clorofila, porém, promoveu aumento dos compostos fenólicos em Achillea collina.

TABELA 1. Teor de flavanóides $\left(\mathrm{mg} 100 \mathrm{~g}^{-1}\right)$, vitamina $\mathrm{C}\left(\mathrm{mg} 100 \mathrm{~g}^{-1}\right)$, fenois totais $(\mathrm{mg} 100$ $\left.\mathrm{g}^{-1}\right)$, Concentração eficiente $\left(\mathrm{CE}_{50} \mathrm{mg} / \mathrm{mL}\right)$ e índice de atividade antioxidante em folhas de jambu (cv. Jambuarana), cultivado sob adubação orgânica e mineral

\begin{tabular}{|c|c|c|c|c|c|c|}
\hline \multirow{2}{*}{$\begin{array}{c}\text { Tipos de } \\
\text { Adubação }\end{array}$} & \multirow{2}{*}{ Colheita } & Flavonoides & Vitamina C & Fenois & $\mathrm{CE}_{50}$ & IAA \\
\hline & & \multicolumn{5}{|c|}{ Folha } \\
\hline Orgânico & \multirow{2}{*}{$1^{\circ}$} & $2,05 b$ & $1,98 \mathrm{a}$ & $310,00 \mathrm{a}$ & $90,50 \mathrm{~b}$ & $1,37 \mathrm{~b}$ \\
\hline Mineral & & $2,60 \mathrm{a}$ & $1,48 b$ & $320,00 \mathrm{a}$ & $85,90 \mathrm{~b}$ & $1,40 \mathrm{~b}$ \\
\hline Orgânico & \multirow{2}{*}{$2^{\circ}$} & $1,97 \mathrm{c}$ & $1,28 \mathrm{c}$ & $360,00 \mathrm{a}$ & $68,50 \mathrm{c}$ & $1,72 \mathrm{a}$ \\
\hline Mineral & & $2,29 \mathrm{a}$ & $1,30 \mathrm{c}$ & $270,00 \mathrm{a}$ & $109,80 a$ & $1,07 \mathrm{c}$ \\
\hline CV (\%) & & 15,98 & 4,30 & 0,63 & 17,79 & 19,27 \\
\hline
\end{tabular}

$\mathrm{CE}_{50}=$ Concentração eficiente; $\mathrm{IAA}$ = índice de atividade antioxidante

TABELA 2. Teor de flavonoides $\left(\mathrm{mg} 100 \mathrm{~g}^{-1}\right)$, vitamina $\mathrm{C}\left(\mathrm{mg} 100 \mathrm{~g}^{-1}\right)$, fenois totais $(\mathrm{mg} 100$ $\left.\mathrm{g}^{-1}\right)$, Concentração eficiente $\left(\mathrm{CE}_{50} \mathrm{mg} / \mathrm{mL}\right)$ e índice de atividade antioxidante em inflorescência de jambu (cv. jambuarana), cultivado sob adubação orgânica e mineral

\begin{tabular}{|c|c|c|c|c|c|c|}
\hline \multirow{2}{*}{$\begin{array}{r}\text { Tipos de } \\
\text { Adubação }\end{array}$} & \multirow{2}{*}{ Colheita } & \multirow{2}{*}{\multicolumn{5}{|c|}{$\begin{array}{cr}\text { Vitamina C } & \text { Fenois } \\
\text { Inflorescência }\end{array}$}} \\
\hline & & & & & & \\
\hline Orgânico & \multirow{2}{*}{$1^{\circ}$} & $4,16 \mathrm{c}$ & $2,36 \mathrm{a}$ & $340,00 \mathrm{~b}$ & $81,82 \mathrm{~b}$ & $1,48 \mathrm{a}$ \\
\hline Mineral & & $5,00 a$ & $1,90 \mathrm{~b}$ & $300,00 \mathrm{~b}$ & $82,18 \mathrm{c}$ & $1,44 b$ \\
\hline Orgânico & \multirow{3}{*}{$2^{\circ}$} & $4,13 \mathrm{c}$ & $1,39 c$ & $320,00 \mathrm{~b}$ & $106,14 \mathrm{a}$ & $1,11 \mathrm{c}$ \\
\hline Mineral & & $4,77 \mathrm{~b}$ & $1,38 \mathrm{c}$ & $460,00 \mathrm{a}$ & $82,29 \mathrm{c}$ & $1,43 \mathrm{~b}$ \\
\hline CV (\%) & & 6,99 & 4,81 & 10,41 & 13,39 & 16,22 \\
\hline
\end{tabular}


Para o teor de vitamina $\mathrm{C}$ em folhas e inflorescências de jambu, houve diferença significativa na primeira colheita, onde a adubação orgânica foi superior a adubação mineral, apresentando valores de $1,98 \mathrm{mg} 100 \mathrm{~g}^{-1}$ nas folhas e 2,36 mg $100 \mathrm{~g}^{-1}$ nas inflorescências. Na segunda colheita não foi observado efeito significativo entre as adubações, tanto para folhas como para inflorescências (Tabelas 1 e 2). Assim, nota-se que a vitamina $\mathrm{C}$ ocorreu em maior teor somente na primeira colheita de plantas de origem orgânica, logo o teor de vitamina C parece ser dependente da adubação orgânica, quando se analisa folha de jambu. REMBIAŁKOWSKA (2007) realizando estudo na qualidade de produtos orgânicos verificou que esse tipo de produção induz menores teores de nitrato e resíduos de pesticida e maior teor de vitamina $C$, aminoácidos essenciais e açúcar total. Outros autores também relataram aumento de vitamina $\mathrm{C}$ em espécies hortícolas orgânicas, como em couve chinesa, alface e repolho (ISMAIL \& FUN, 2003) e tomates (CHASSY et al., 2006), em comparação as convencionais. Segundo CARVALHO \& MULLER (1996), a planta de jambu é rica em elementos nutritivos como ferro, e ainda possui as vitaminas $B 1, B 2$, niacina, vitamina $C$, vitamina $A$ e cálcio. $O$ jambu apresenta valor nutritivo por 100 gramas de folhas de $89,0 \mathrm{~g}$ de água, valor energético de aproximadamente 32,0 calorias. Contém em cada $100 \mathrm{~g}: 1,9 \mathrm{~g}$ proteínas, 0,3 $\mathrm{g}$ de lipídios; 7,2 $\mathrm{g}$ de carboidratos, 1,3 $\mathrm{g}$ de fibras, 1,6 $\mathrm{g}$ de cinzas, $162,0 \mathrm{mg}$ de cálcio, $41 \mathrm{mg}$ de fósforo, 4,0mg de ferro; 0,03 $\mathrm{mg}$ de vitamina B10,21 $\mathrm{mg}$ de vitamina B2, 1,0 mg de niacina e $20,0 \mathrm{mg}$ de vitamina C (VILLACHICA et al., 1996).

Para o CE50 a adubação orgânica foi superior a adubação mineral na primeira colheita para inflorescência e na segunda colheita para folhas (Tabelas $1 \mathrm{e}$ 2). Isso pode ser constatado pelo resultado da análise do índice de atividade antioxidante (IAA), que é um índice mais prático para determinar o potencial antioxidante de um sistema (SCHERER \& GODOY, 2009), devido as dificuldades de interpretação do CE50. Os valores encontrados neste estudo, de acordo com SHERER \& GODOY (2009) demonstraram que as folhas e inflorescências possuem atividade antioxidante forte, já que os valores encontrados estão entre 1 e 2 . Outras pesquisas relacionam o cultivo orgânico com 0 incremento de substâncias com potencial antioxidante, benéficas a saúde humana (LIMA et al., 2008, LIMA \& VIANELLO, 2011, ROSSETTO et al., 2009). A porcentagem de atividade antioxidante (\%AA) corresponde a quantidade de DPPH consumida pelo antioxidante, sendo que a quantidade de antioxidante necessaria para decrescer a concentracao inicial de DPPH em $50 \%$ é denominada concentração eficiente (CE50), tambem chamada de concentracao inibitoria (Cl50). Quanto maior o consumo de DPPH por uma amostra, menor sera a sua CE50 e maior a sua atividade antioxidante (SOUSA et al., 2007).

Os coeficientes de correlações de Pearson (Tabela 3) entre as adubações utilizadas e as análises realizadas em folhas e inflorescências de plantas de jambu mostram correlação forte e positiva para adubação orgânica para as características de vitamina $C$ inflorescência $\left(0,840^{*}\right)$ e concentração eficiente na inflorescência $\left(0,986^{\star}\right)$, com significância de $5 \%$. Já a adubação mineral apresentou correlação moderada para teor de flavonoides nas folhas $\left(0,489^{*}\right)$, teor de fenois nas inflorescências $\left(0,784^{*}\right)$, enquanto que concentração eficiente também nas inflorescências $\left(0,990^{*}\right)$ mostrou forte correlação. FALLER \& FIALHO (2009) verificando a correlação de Pearson em cenoura, batata, cebola, brócolis e repolho orgânicos e convencionais, constataram que os polifenois em geral, mostraram alta correlação com a capacidade antioxidante dos vegetais estudados, independentes 
da adubação aplicada. No presente estudo, a adubação, de acordo com a correlação de Pearson, orgânica influenciou fortemente os níveis de vitamina C, enquanto a adubação mineral influenciou o $\mathrm{CE}_{50}$.

TABELA 3. Correlação de Pearson em folha e inflorescência de jambu (cv. jambuarana), sob adubação orgânica e mineral

\begin{tabular}{cccccc}
\hline $\begin{array}{c}\text { Tipo de } \\
\text { adubação }\end{array}$ & $\begin{array}{c}\text { Flav. } \\
\left(\mathrm{mg} \mathrm{mL}^{-1}\right)\end{array}$ & $\begin{array}{c}\text { Vit. C } \\
(\mathrm{mg} / 100 \mathrm{~g})\end{array}$ & Fenois & $\begin{array}{c}\mathrm{CE}_{50} \\
(\mathrm{mg} / \mathrm{mL})\end{array}$ & IAA \\
\cline { 2 - 6 } & \multicolumn{5}{c}{ Folha } \\
\hline Mineral & $0,489^{*}$ & $0,607 \mathrm{~ns}$ & $0,786 \mathrm{~ns}$ & $0,524 \mathrm{~ns}$ & $0,786 \mathrm{~ns}$ \\
Orgânica & $0,368 \mathrm{~ns}$ & $0,600 \mathrm{~ns}$ & $0,760 \mathrm{~ns}$ & $0,726 \mathrm{~ns}$ & $0,578 \mathrm{~ns}$ \\
\hline & \multicolumn{5}{c}{ Inflorescência } \\
Mineral & $0,561 \mathrm{~ns}$ & $0,753 \mathrm{~ns}$ & $0,784^{*}$ & $0,990^{*}$ & $0,617 \mathrm{~ns}$ \\
Orgânica & $0,189 \mathrm{~ns}$ & $0,840^{*}$ & $0,562 \mathrm{~ns}$ & $0,986^{*}$ & $0,436 \mathrm{~ns}$ \\
\hline
\end{tabular}

${ }^{*}$ Correlação foi significativa em nível de $5 \%(\mathrm{P}<0,05) ; \mathrm{IAA}=$ índice de atividade antioxidante; $\mathrm{CE}_{50}=$ Concentração eficiente; Fenois = fenois; Flav.= flavonoides; Vit. $\mathrm{C}=$ vitamina $\mathrm{C}$.

Muitos estudos têm mostrado que os polifenois são importantes moléculas que agem como antioxidantes, muitas vezes são consideradas uma das principais fontes de antioxidantes naturais em vegetais (SLAVIN et al., 2009). Assim, a atividade antioxidante analisada a partir de três metodologias (medida da atividade do sequestro do radical livre por DPPH, por Folin-Ciocalteau e pelo método enzimático) apresentou diferenças entre si (Figura 1).

A metodologia enzimática parece ter sido mais eficiente para extração e medida do teor de polifenois em alguns tratamentos, como apresentou poder extrator para compostos fenólicos das inflorescências convencionais de jambu na primeira como na segunda colheita e para folha orgânica na segunda colheita, conforme indicado na Figura 1.

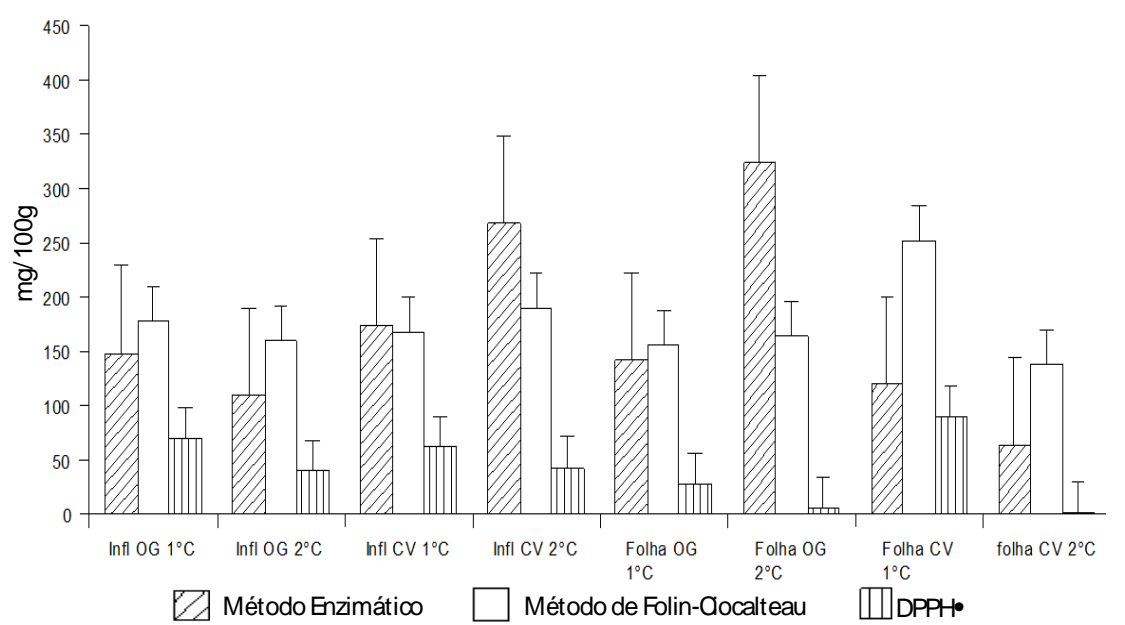

Infl OG $1^{\circ} \mathrm{C}=$ Inflorescência orgânica $1^{\circ}$ colheita; Infl $O G 2^{\circ} \mathrm{C}=$ Inflorescência orgânica $2^{\circ}$

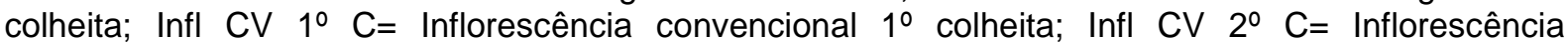
Convencional $2^{\circ}$ colheita; Folha OG $1^{\circ} \mathrm{C}=$ Folha orgânica $1^{\circ}$ colheita; Folha OG $2^{\circ} \mathrm{C}=$ Folha orgânica $2^{\circ}$ colheita; Folha CV $1^{\circ} \mathrm{C}=$ Folha Convencional $1^{\circ}$ colheita; Folha CV $2^{\circ} \mathrm{C}=$ Folha Convencional $2^{\circ}$ colheita.

FIGURA 1. Polifenois em folhas e inflorescência de jambu (cv. Jambuarana), cultivado sob adubação orgânica e mineral, através de três métodos bioquimicos. 
Entre os métodos químicos aplicados para determinar a capacidade antioxidante de um composto, o método DPPH é um dos mais utilizados, por ser considerado prático, rápido e estável (ESPIN et al., 2000). No entanto, nessa pesquisa foi a metodologia que mostrou menor concentração dos polifenois para folhas e inflorescências na adubação orgânica e mineral, tanto na primeira como na segunda colheita de jambu (Figura 1). Provavelmente, esse efeito se deve a solubilidade das substâncias medidas pelo método. O DPPH pode reagir com compostos fenólicos, bem como com ácidos aromáticos contendo apenas um grupamento (SANTOS et al., 2007). Segundo esses mesmos autores o método de DPPH é muito utilizado para se determinar a atividade antioxidante em extratos e substancias isoladas como compostos fenólicos, fenilpropanoides, fenólicos totais, flavonoides, o que justificaria essa menor concentração de polifenois totais na amostra de jambu quando analisada via DPPH.

\section{CONCLUSÃO}

A adubação mineral é eficaz, quanto aos teores de flavonoides em jambu, sendo as folhas o principal local de produção e foi eficaz também na produção de fenois totais em inflorescências. A vitamina $C$ ocorreu em maior teor somente na primeira colheita de plantas de origem orgânica. $O$ uso do método enzimático é eficiente para determinação de polifenois em jambu.

\section{AGRADECIMENTOS}

Os autores agradecem a CAPES pela concessão da bolsa de mestrado à primeira autora e ao CNPq pelo financiamento.

\section{REFERENCIA}

AWAD, M. A.; de JAGER, A.; WESTING, L. M. Flavonoid and chlorogenic acid levels in apple fruit: characterisation of variation. Scientia Horticulturae, v. 83, n. 3-4, p. 249-263, 2000. Doi:10.1016/S0304-4238(99)00124-7

BERNARDES, N. R.; PESSANHA, F. F.; OLIVEIRA, D. B. Alimentos Funcionais: Uma breve revisão. Ciência e Cultura - Revista Científica Multidisciplinar do Centro Universitário da FEB, v.6, n. 2. Novembro, 2010.

BORGES, L. S.; GOTO, R.; LIMA, G. P. P. Índices morfo-fisiológicos e produtividade de cultivares de jambu influenciadas pela adubação orgânica e mineral. Biosci. J., Uberlândia, v. 30, n. 6, p. 1768-1778, 2014a

BORGES, L.S.; NUNES, K.N.M.; JACQUES, R.A. LIMA, G.P.P. Perfil cromatográfico do óleo essencial de jambu identificados por cromatógrafo a gás acoplado a espectrômetro de massas. Cultivando o Saber. V 7 - n³, p. 254 - 266, 2014b

BORGES, L.S. GUERRERO, A.G.; GOTO, R.; LIMA, G.P.P. Produtividade e acúmulo de nutrientes em plantas de jambu, sob adubação orgânica e mineral. Semina: Ciências Agrárias, v. 34, n. 1, p. 83-94, 2013. . DOI: http://dx.doi.org/10.5433/16790359.2013v34n1p83.

BORGES, L. S., GUERRERO, A. C., GOTO, R., LIMA, G. P. P. Exportação de nutrientes em plantas de jambu, sob diferentes adubações. Semina: Ciências 
Agrárias, Londrina, v. $34, \quad$ n. $1, \quad$ p. 107-116. 2013b. DOI: http://dx.doi.org/10.5433/1679-0359.2013v34n1p107

BORGES, L.S. VIEIRA, M.A.R. ; MARQUES, M.O.M. ; VIANELLO, F. ; LIMA,G.P.P . Influence of organic and mineral soil fertilization and essential oil of Spilanthes oleracea. American Journal of Plant Physiology, v.7, n. 3, p. 135-142, 2012. DOI: 10.3923/ajpp.2012.135.142

BRAND-WILLIAMS, W.; CUVELIER, M. E.; BERSET, C. Use of free radical method to evaluate antioxidant activity. LWT-Food Science and Technology, v. 28, n. 1, p. 25-30, 1995.

CARVALHO, J. E. U. de; MÜLLER, C. H. Jambu, Spilanthes oleracea L.. In: VILLACHICA, H., CARVALHO, J. E. U. de; MÜLLER, C. H.; DÍAZ, C.; ALMANZA, M. Frutales y hortalizas. promissorios de la Amazônia. Lima: Tratado de Coperacion Amazônica, 1996. p.32 1-327.

CHASSY, A. W. BUI L, RENAUD, E.N.C., HORN, M.V., MITCHELL, A... Three-year comparison of the content ofantioxidant microconstituents and several quality characteristics in organic and conventionally managed tomatoes and bell peppers. Journal Agriculture Food Chemistry, v. 54, n. 21, p. 8244-8252, 2006.

DIXON, R. A.; PAIVA, N. L. Stress-induced phenylpropanoid metabolism. Plant Cell, v.7, n. 7, p. 1085-1097. 1995.

ESPINDOLA, C. R., TOSIN, W. A. C., PACCOLA, A. A. Levantamento pedológico da Fazenda Experimental São Manuel. In: CONGRESSO BRASILEIRO DE CIÊNCIA DO SOLO, 14., 1973, Santa Maria. Anais... Santa Maria: Sociedade Brasileira de Ciência do Solo, p. 650-651. 1974.

ESPIN, J. C.; SOLER-RIVAS, C.; WICHERS, H. J. Characterization o the total free radical scavenging capacity of vegetable oils and oil fractions using 2,2-diphenyl-1picrylhydrazyl radical. Journal of Agricultural and Food Chemistry. v.48, n. 3, p.648-656, 2000.

FALLER, A. L. K.; FIALHO, E. The antioxidant capacity and polyphenol content of organic and conventional retail vegetables after domestic cooking. Food Research International, v. 42, n. 1, p. 210-215, 2009.

GIORGI, A.; MINGOZZI, M.; MADEO, M. ; SPERANZA, G.; COCUCCI, M. Effect of nitrogen starvation on the phenolic metabolism and antioxidant properties of yarrow (Achillea collina Becker ex Rchb.). Food Chemistry, v.114, n 1, p. 204-21, 2009. DOI: 10.1016/j.foodchem.2008.09.039.

HOMA, A. K. O.; SANCHES, R. S.; MENEZES, A. J. E. A.; GUSMÃO, S. A. L. Etnocultivo do Jambu para abastecimento da cidade de Belém, estado do Pará. Amazônica: Ciência \& Desenvolvimento, Belém, v. 6, n. 12, p.125-141, 2011.

ISMAIL, A.; FUN, C. S. Determination of vitamin C, b-carotene and riboflavin contents in five green vegetables organically and conventionally grow. Malaysian 
Journal Nutrition, v. 9, n. 1, p. 31-39, 2003.

JANSSEN, M.; HAMM, U. Product labelling in the market for organic food: Consumer preferences and willingness-to-pay for different organic certification logos. Food Quality and Preference, v. 25, n. 1 p. 9-22, 2012.

LEE, K. W. KIM, Y. J.; LEE, H. J.; LEE, C. Y. Cocoa has more phenolic phytochemicals and a higher antioxidant capacity than teas and red wine. Journal of Agriccultural and Food Chemmistry, v. 51, n. 25, p. 7292-7295, 2003. DOI: $10.1021 / \mathrm{jf} 0344385$

LI-CHEN, W. Fan, N. C., Lin, M. H., Chu, I. R., Huang, S. J., Hu, C. Y., \& Han, S. Y.Anti-inflammatory Effect of Spilanthol from Spilanthes acmella on Murine Macrophage by Down-Regulating LPS-Induced Inflammatory Mediators. Journal of Agricultural and Food Chemitry, v. 56, n. 7, p.2341-2349, 2008. DOI: $10.1021 /$ jf073057e.

Lima, G. P. P. ; MACHADO, TATIANA MARQUINI ; Oliveira, Luciana Manoel de ; BORGES, LUCIANA DA SILVA ; PEDROSA, VALBER DE ALBUQUERQUE ; VANZANI, PAOLA ; Vianello, Fabio . Ozonated water and chlorine effects on the antioxidant properties of organic and conventional broccoli during postharvest. Scientia Agricola (USP. Impresso), v. 71, p. 151-156, 2014. Doi.org/10.1590/S010390162014000200010

LIMA, G. P. P. LOPES, T. V. C. ; ROSSETTO, M. R. M.; VIANELLO, F. Nutritional composition, phenolic compounds, nitrate content in eatable vegetables obtained by conventional and certified organic grown culture subject to thermal tretment. International Journal of Food Science and Technology, v. 44, n. 6, p. 1118-1124, 2009. DOI: 10.1111/j.1365-2621.2009.01928.x

LIMA, G. P. P. DA ROCHA, S. A. ; TAKAKI, M. ; RAMOS, P. R. R. ; ONO, E. O. Comparison of polyamine, phenol and flavonoid contents in plants grown under conventional and 23 organic methods. International Journal of Food Science Technology, v. $43, \quad$ n. $10, \quad$ p. $1838-1843,2008$. DOI: $10.1111 / \mathrm{j} .1365-$ 2621.2008.01725.x

LIMA, G.P.P.; VIANELLO, F. Review on the main differences between organic and conventional plant-based foods. International Journal of Food Science and Technology, v. 46, n. 1, p. 1-13, 2011. DOI: 10.1111/j.1365-2621.2010.02436.x

LORENZI, H.; MATOS, F. J. A. Plantas medicinais do Brasil: nativas e exóticas cultivadas. Nova Odessa, SP: Instituto Plantarum, 2002. 396 p.

MAPA-Ministério da Agricultura, Pecuária e Abastecimento (Mapa). Disponível em: $\quad$ <http://www.agricultura.gov.br/desenvolvimento-sustentavel/organicos>. Acessado em 12/10/2012.

PICCHI, V. MIGLIORI, C., SCALZO, R. L., CAMPANELLI, G., FERRARI, V., DI CESARE, L. F. Phytochemical content in organic and conventionally grown Italian cauliflower. Food Chemistry, v. 130, n. 3, p. 501-509, 2012. 
Doi:10.1016/j.foodchem.2011.07.036

POPOVA, M. BANKOVA, V., BUTOVSKA, D., PETKOV, V., NIKOLOVADAMYANOVA, B., SABATINI, A.G., MARCAZZAN, G.L., BOGDANOV, S. Validated methods for the quantification of biologically active constituents of poplar-type propolis58. Phytochemical Analysis, v. 15, n. 4, p. 235-240, 2004. DOI: $10.1002 /$ pca. 777

REMBIAŁKOWSKA, E. Quality of plant products from organic agriculture. Journal of the Science of Food and Agriculture, v. 87, n. 15, p. 2757-2762, 2007. DOI: $10.1002 /$ jsfa.3000

ROSSETTO, M. R. M. VIANELLO, F. ; ROCHA, S. A. ; LIMA, G. P. P. Antioxidant substances and pesticide in parts of beet organic and conventional manure. African Journal of Plant Science, v.3, n. 11, p. 245-253, 2009.

ROSSETO, M. R. M. ; FABIO, VIANELLO ; SAEKI, M. J. ; LIMA, G. P. P. Polyamines in conventional and organic vegetables exposed to exogenous ethylene. Food Chemistry, v. 188, p. 212-220, 2015. doi:10.1016/j.foodchem.2015.04.125

SANTOS, M. H dos; BATISTA, B.L. ; DUARTE, S.M.S; ABREU, C.M.P.A; GOUVÊA, C. M. C. P. Influência do processamento e da torrefação sobre a atividade antioxidante do café (Coffea arabica). Química Nova. v.30, n. 3, p. 604-610, 2007.

SCHERER, R.; GODOY, H. T. Antioxidant activity index (AAI) by the 2,2-diphenyl-1picrylhydrazyl method. Food Chemistry, v. 112, N. 3, p. 654-658, 2009. Doi:10.1016/j.foodchem.2008.06.026

SINGLETON, V. L.; ROSSI-Jr, J.A. Colorimetry of total phenolics with phosphomolybdic-phosphotungstic acid reagents. American Journal of Enology and Viticulture. v.16, n. 3, p. 144-158, 1965.

SGANZERLA, E., MARTINS, R.M., SINGH, D. Alimentos orgânicos no Brasil: história, cultura e gastronomia. Esplendor: Curitiba. 2013. 208p.

SLAVIN, M. Cheng, Z., Luther, M., Kenworthy, W., Yu, L. L.. Antioxidant properties and phenolic, isoflavone, tocopherol and carotenoid composition of maryland-grown soybean lines with altered fatty acid profiles. Food Chemistry, v.114, n. 1, p. 20-27, 2009. Doi:10.1016/j.foodchem.2008.09.007.

SMOLEN, S.; SADY, W. The effect of various nitrogen fertilization and foliar nutrition regimes on the concentrations of sugars, carotenoids and phenolic compounds in carrot (Daucus carota L.). Scientia Horticulturae, Krakow, Poland, v. 120, n. 3, p. 315-324, 2009. Doi:10.1016/j.scienta.2008.11.029.

SOUSA, C. M.; SILVA, H. R.; VIEIRA-JR, G. M.; AYRES, M. C. C.; COSTA, C. D.; ARAÚJO, D. S.; CHAVES, M. H.. Fenois totais e atividade antioxidante de cinco plantas medicinais. Química Nova. v. 30, n. 2, p. 351-355, abr. 2007.

TERADA, M. WATANABE, Y., KUNITOMO, M., HAYASHI, E.. Differential rapid ENCICLOPÉDIA BIOSFERA, Centro Científico Conhecer - Goiânia, v.11 n.22; p. 799 2015 
analysis of ascorbic acid and ascorbic acid 2-sulfate by dinitrophenylhy drazine methol. Analytical Biochemistry, v. 84, n. 2, p. 604-608, 1978. Doi:10.1016/00032697(78)90083-0

VILLACHICA, H. ALEM ROJO, A., WINOGRAD, M., FARROW, A., EADE, J., KOOLEN, R., SALDOVAL, $H$. Frutales y Hortalizas Promissórios de La Amazônia. Lima: Tratado de Cooperacion Amazônica. Secretaria-Pro-tempore, 1996. 110 p. (TCA-SPT, 44).

ZENNARO, L.; ROSSETTO, M.; VANZANI, P.; DE MARCO, V.; SCARPA, M.; BATTISTIN, L.; RIGO, A. A method to evaluate capacity and efficiency of water soluble antioxidants as peroxyl radical scavengers. Archives of biochemistry and biophysics, 462(1), 38-46. 2007. Doi:10.1016/j.abb.2007.03.017. 